\title{
Recognition of heparin-induced thrombocytopenia and initiation of argatroban therapy after cardiothoracic surgery in the intensive care unit
}

\author{
Linda J. Demma, MD, PhD, ${ }^{\text {a }}$ Christopher A. Paciullo, PharmD, BCPS, ${ }^{b}$ and Jerrold H. Levy, MD, FAHA ${ }^{\mathrm{a}}$
}

Objective: Patients recovering from cardiothoracic surgery are known to be at increased risk of heparin-induced thrombocytopenia. Postoperatively, if heparin-induced thrombocytopenia is suspected, heparin is discontinued immediately and an alternative anticoagulant, such as the direct thrombin inhibitor argatroban, is administered. Current data regarding the safety and efficacy of argatroban in the postoperative cardiothoracic surgical patient in the intensive care setting are limited.

\begin{abstract}
Methods: Data were collected retrospectively from January 1, 2007, to December 31, 2010, from patients tested for antiplatelet factor 4/heparin antibodies on clinical suspicion of heparin-induced thrombocytopenia after cardiothoracic surgery. We evaluated the use of argatroban as a therapeutic agent for the postoperative treatment of suspected heparin-induced thrombocytopenia by comparing thrombotic and bleeding events, platelet dynamics, antiplatelet factor 4/heparin antibody titer, and clinical probability score between patients who did and did not receive argatroban.
\end{abstract}

Results: Eighty-seven patients were included; 47 patients (54\%) were treated with argatroban, and 40 patients $(46 \%)$ were not treated with argatroban. There was no association between argatroban therapy and bleeding, mortality, length of stay, or pretreatment thrombotic events. Among all patients, antiplatelet factor $4 /$ heparin antibody titer and clinical probability score were higher in patients treated with argatroban.

Conclusions: Clinical suspicion of heparin-induced thrombocytopenia as detected by clinical probability score and thrombotic complications should prompt immediate cessation of heparin and initiation of an alternative anticoagulant such as argatroban. The results from this study demonstrate that argatroban should be considered without increased risk for adverse events, including bleeding, in the cardiothoracic intensive care unit after surgery. (J Thorac Cardiovasc Surg 2012;143:1213-8)

Patients undergoing cardiothoracic surgery routinely receive large doses of unfractionated heparin during their procedure. Platelet activation during surgery and cardiopulmonary bypass (CPB) likely confers an increased risk for postoperative heparin-induced thrombocytopenia (HIT), which occurs in $1 \%$ to $3 \%$ of these patients. ${ }^{1-3}$ Patients who develop HIT antibodies, antibodies that recognize multimeric platelet factor 4 (PF4)/heparin complexes on platelet surfaces, are considered to be in a procoagulant state and at high risk for adverse thrombotic complications. ${ }^{4}$ Heparin-induced thrombocytopenia with thrombosis (HITT) is the clinical syndrome in which patients with HIT antibodies develop myocardial infarction, pulmonary embolism (PE), limb

\footnotetext{
From the Departments of Anesthesiology and Critical Care, ${ }^{\mathrm{a}}$ Emory University School of Medicine and Emory Healthcare, Atlanta, Ga; and the Department of Pharmaceutical Services, ${ }^{\text {b }}$ Emory Healthcare, Atlanta, Ga.

Funding: Financial support was provided by GlaxoSmithKline and Emory University Department of Anesthesiology.

Disclosures: Authors have nothing to disclose with regard to commercial support.

Received for publication Dec 20, 2010; revisions received June 5, 2011; accepted for publication July 19, 2011; available ahead of print Feb 13, 2012.

Address for reprints: Jerrold H. Levy, MD, FAHA, Emory University Hospital, 1364

Clifton Road, Atlanta, GA 30322 (E-mail: jlevy01@emory.edu).

0022-5223/\$36.00

Copyright (C) 2012 by The American Association for Thoracic Surgery

doi:10.1016/j.jtcvs.2011.07.068
}

ischemia, or deep vein thromboses (DVTs) secondary to HIT. $^{5-7}$ Postoperatively, if HIT is suspected, heparin is discontinued immediately and an alternative anticoagulant such as the direct thrombin inhibitor (DTI) argatroban is administered, either as a prophylactic anticoagulant or for the treatment of thrombosis in patients with HITT. ${ }^{8}$ There is a high index of suspicion for HIT in the cardiothoracic intensive care unit (ICU) because of evidence that this patient population is at increased risk of HITT.

Argatroban's advantage in a critically ill patient is that it has a relatively short half-life and is generally not affected by renal dysfunction, gender, or age. ${ }^{9,10}$ The anticoagulant effects of argatroban are monitored by measuring the activated partial thromboplastin time (aPTT), which increases in a dose-dependent manner, parallel to the levels of argatroban. ${ }^{11}$ The argatroban dose is adjusted to achieve an aPTT of 60 to 80 seconds or an aPTT of 1.5 to 3 times the patient's baseline aPTT. ${ }^{12,13}$ It is standard practice in our cardiothoracic ICU to begin argatroban at first clinical suspicion of HIT based on rapid decline or persistently low platelet counts $(\Delta \mathrm{p})$ postoperatively. Argatroban is begun immediately on suspicion of HIT and continued if a PF4/ heparin immunoassay is positive; argatroban is typically discontinued if the PF4/heparin immunoassay is negative. 


$$
\begin{aligned}
& \text { Abbreviations and Acronyms } \\
& \begin{aligned}
\text { aPTT } & =\text { activated partial thromboplastin time } \\
\text { CPB } & =\text { cardiopulmonary bypass } \\
\text { DTI } & =\text { direct thrombin inhibitor } \\
\text { DVT } & =\text { deep vein thrombosis } \\
\text { ELISA } & =\text { enzyme-linked immunosorbent assay } \\
\text { HIT } & =\text { heparin-induced thrombocytopenia } \\
\text { HITT } & =\text { heparin-induced thrombocytopenia } \\
& \text { with thrombosis } \\
\text { ICU } & =\text { intensive care unit } \\
\text { Ig } & =\text { immunoglobulin } \\
\text { OD } & =\text { optical density } \\
\Delta \mathrm{p} & =\text { platelet count } \\
\text { PE } & =\text { pulmonary embolism } \\
\text { PF4 } & =\text { platelet factor } 4 \\
\text { SRA } & =\text { serotonin release assay }
\end{aligned}
\end{aligned}
$$

Because cardiac surgical patients are at potential risk for bleeding and other adverse outcomes of postoperative anticoagulation, it is important to maintain clinical suspicion for HIT with the use of a standard pretest clinical probability tool, recognize potential risks and benefits of argatroban therapy in the cardiothoracic surgical patient population, and treat with argatroban when indicated. Although there are previous reports using argatroban as an anticoagulant, there is little information about its use postoperatively in the critical care setting after cardiac surgery. ${ }^{14,15}$ We evaluated our experience with argatroban as a therapeutic agent for the postoperative treatment of suspected HIT in the cardiothoracic ICU. We investigated thrombotic and bleeding events, laboratory markers of HIT including platelet dynamics and PF4/ heparin immunoassay results, and clinical probability scores in patients who did and did not receive argatroban.

\section{MATERIALS AND METHODS Patients}

After institutional approval, 3508 patients admitted to the cardiothoracic ICU after cardiothoracic surgery (cardiac cases, and heart and lung transplants) from January 1, 2007, to December 31, 2010, were screened for inclusion. Data were collected by retrospective medical chart review from patients who had been tested for anti-PF4/heparin antibodies on clinical suspicion of HIT within 30 days after surgery. The clinical suspicion of HIT was determined on the basis of a persistently low or rapid decline in $\Delta \mathrm{p}$, or new evidence of thrombosis after surgery.

HIT antibody testing was performed by the Special Hemostasis Laboratory at Emory University Hospital. Plasma samples from January 2007 to August 2009 were tested using a commercial enzyme-linked immunosorbent assay (ELISA) for antibodies of immunoglobulin (Ig)-G, IgA, and IgM classes (PF4 Enhanced; GTI Diagnostics, Waukesha, Wis) as previously described. ${ }^{16}$ Plasma samples collected from September 2009 to December2010 were tested using a commercial ELISA specific for IgG antibodies (Zymutest HIA IgG; HYPHEN BioMed, Aniara, Mason, Ohio). For both assays, results were expressed in optical density (OD) units; a value of 0.4 or greater was considered positive because the negative predictive value for
OD less than 0.4 in both assays is known to be $100 \% .{ }^{17}$ When possible, the serotonin release assay (SRA), which is currently considered the gold standard for the laboratory diagnosis of HIT, was performed on samples demonstrating an OD of 0.4 or greater by a designated reference laboratory at the discretion of the ordering physician. A result of $20 \%$ or greater serotonin release was reported as positive by the reference laboratory.

The indication for anticoagulation in patients with suspected HIT was a thrombotic complication, rapid decline, or persistently low $\Delta \mathrm{p}$. Argatroban was initiated as a continuous infusion of $1.5 \mu \mathrm{g} / \mathrm{kg} / \mathrm{min}$ for patients with normal liver function. A reduced dose of $0.5 \mu \mathrm{g} / \mathrm{kg} / \mathrm{min}$ was used if the patient had liver dysfunction, defined as a Child-Pugh score greater than $7 .{ }^{18}$ Per protocol, the aPTT was measured 2 hours after the start of the argatroban infusion, and dose adjustments were made to maintain desired aPTT levels (1.5-3 times baseline, not to exceed 100 seconds). The aPTT was assessed 4 hours after any dose adjustment or daily for patients with 2 or more therapeutic aPTT levels. Argatroban was avoided if patients were actively bleeding or had evidence of acute stroke or other central nervous system process.

Patients were divided into 2 groups: those who received anticoagulation with argatroban and those who did not. Patients who did not receive argatroban received heparin subcutaneously for DVT prophylaxis or intravenously for a specific anticoagulation indication (eg, history of PE, DVT, valve surgery, or known hypercoagulable state without suspicion of HIT). Anti-Xa levels were used for monitoring intravenous heparin therapy with high- and low-dose titrations. In these patients, 3 to 5 titrations were typically required. If patients were not receiving any anticoagulation, mechanical methods of DVT prophylaxis were used.

Detailed data were collected on age, gender, date of admission and discharge, date and cause of death if applicable, number of doses of argatroban received, surgery date and type, use of $\mathrm{CPB}$, baseline $\Delta \mathrm{p}$ before surgery, daily $\Delta \mathrm{p}$ when available from 2 days before surgery to 30 days postsurgery, and PF4/heparin OD. The postoperative decrease in $\triangle \mathrm{p}$ was defined as the percent decline in platelets from the platelet peak attained postoperatively to the subsequent platelet nadir. Data on adverse outcomes were also collected, including occurrence of thrombotic complications (defined as DVT, myocardial infarction, embolic cerebrovascular accident, PE, or limb ischemia), mortality, length of stay, and bleeding complications.

The clinical features of HIT were quantified using the 4Ts score as described previously. ${ }^{19}$ Briefly, the 4 "Ts" in the 4Ts score are (1) thrombocytopenia with a $\Delta \mathrm{p}$ decrease greater than $50 \%$ and platelet nadir of $\geq 20$; (2) timing of platelet fall with clear onset between days 5 and 10 or platelet decrease $\leq 1$ day (prior heparin exposure within 30 days); (3) new thrombosis (confirmed); and (4) no other likely cause for thrombocytopenia. ${ }^{19}$ The resulting clinical probability scores are divided into high (6-8 points), intermediate (4-5 points), and low ( $\leq 3$ points) groups. In addition, the platelet profiles were categorized as P1, platelet decline greater than $50 \%$ from a postoperative maximum within 5 to 10 days after surgery, or P2, persistent thrombocytopenia beyond 5 days after surgery based on the platelet profiles described by Pouplard and colleagues. ${ }^{20}$

\section{Statistical Analyses}

Patients were divided into 2 groups: those treated with argatroban and those not treated with argatroban. Mean values for length of stay; baseline, minimum, and maximum $\Delta \mathrm{p}$; and $\mathrm{PF} 4 /$ heparin OD were compared between the 2 groups using the Mann-Whitney $U$ test. Categoric variables, including the incidence of mortality, bleeding, thrombotic complications, on-pump surgery, and occurrence of each platelet profile, were compared between the 2 groups with the Fisher exact test (for dichotomous variables). All statistical analyses were performed using SPSS statistics 19.0 (SPSS Inc, Chicago, Ill).

\section{RESULTS \\ Patients}

Eighty-seven patients with an anti-PF4/heparin antibody test conducted within 30 days after cardiothoracic surgery 
(cardiac cases, heart or lung transplants) while in our cardiothoracic ICU were included in this study. Forty-seven patients $(54 \%)$ were treated with argatroban, and 40 patients $(46 \%)$ were not treated with argatroban. Among all patients, $38(46 \%)$ had a platelet decline greater than $50 \%$ from a postoperative maximum within 10 days after surgery consistent with the P1 platelet profile, and 21 patients had persistent thrombocytopenia beyond 5 days after surgery consistent with the $\mathrm{P} 2$ profile. The median $4 \mathrm{Ts}$ score for all patients was 3 (range, 0-7; interquartile range, 1-5).

Among 47 patients treated with argatroban, median 4Ts score was 4 (range, 0-7; interquartile range, 2-5), 22 had a P1 platelet profile, and 15 had a $\mathrm{P} 2$ profile. Mean PF4/heparin OD was 1.012 (range, 0.104-3.000). Six patients had a high 4Ts score, 23 patients had an intermediate 4Ts score, and 18 patients had a low 4Ts score. Thirty-seven (79\%) of the 47 patients treated with argatroban had a positive PF4/ heparin ELISA (OD > 0.4). Of the 10 patients with a negative PF4/heparin ELISA, 5 received only 1 day of treatment with argatroban before it was discontinued when the result of the PF4/heparin ELISA was negative. Five patients with a negative PF4/heparin ELISA continued taking argatroban at the physician's discretion because of a need for continued anticoagulation (history of DVT, PE, limb ischemia, or known hypercoagulable state). SRA was positive in 7 of the 22 patients whose samples were sent for SRA testing.

Recovery of $\Delta \mathrm{p}$ after argatroban was variable; no specific pattern of platelet recovery was seen in either treatment group, likely because of all of the different factors that determine postoperative thrombocytopenia after cardiac surgery. In 32 patients $(68 \%)$, there were transient aPTT values greater than 100 seconds after argatroban was initiated, but all elevated aPTT values resolved with appropriate titration.

Of the 40 patients not treated with argatroban, median 4Ts score was 2 (range, 0-6), 16 had a P1 profile, and 6 had a P2 profile. Thirteen $(33 \%)$ of the 40 patients had a PF4/heparin OD greater than 0.40 (mean, 0.354; range, $0.031-2.365$ ). One patient had a high 4 Ts score, 8 patients had an intermediate 4Ts score, and 31 patients had a low 4Ts score. Seven total samples were sent for SRA testing and 6 were negative, likely the explanation for lack of argatroban initiation. Subcutaneous heparin was withdrawn in the patient with a positive SRA, and alternative anticoagulation was not begun. Of the 13 patients with a positive PF4/ heparin ELISA, subcutaneous heparin for DVT prophylaxis or intravenous heparin for anticoagulation was continued in those who had a negative SRA $(n=6)$, patients were switched from subcutaneous heparin for DVT prophylaxis to enoxaparin or fondaparinux $(n=4)$, or all heparin was discontinued $(\mathrm{n}=3)$. Intravenous heparin was given in 8 of the 40 patients who did not receive argatroban in response to a continued need for intravenous coagulation; all of these patients had a negative PF4/heparin ELISA or SRA.

\section{Complications}

Among the 47 patients treated with argatroban, $11(26 \%)$ died and $3(6 \%)$ had bleeding complications. Two patients had gastrointestinal bleeds likely secondary to peptic ulcer more than 3 weeks after argatroban therapy; 1 patient had epistaxis, hypotension, and oxygen desaturation approximately 2 days after argatroban therapy and died of respiratory failure. In this patient, aPTT had returned to normal, suggesting death was unrelated to argatroban. Among the 11 argatroban-treated patients who died, cause of death was determined to be respiratory failure and oxygen desaturation $(n=2)$, multiorgan system failure $(n=7)$, disseminated intravascular coagulation $(\mathrm{n}=1)$, and cerebrovascular accident with myocardial infarction $(n=1)$. None of the patients who died were receiving argatroban at the time of death. Of 20 total thrombotic complications, the thrombotic event was the clinical sign that prompted HIT testing and argatroban initiation in 15 patients. The remaining 5 additional thrombotic complications occurred despite argatroban.

Among the 40 patients not treated with argatroban, 8 $(20 \%)$ died and $5(13 \%)$ had bleeding complications. Among the 5 patients with bleeding complications that were not treated with argatroban, 1 patient was receiving heparin intravenously for a known hypercoagulable state and 4 patients were receiving heparin $(n=3)$ or fondaparinux $(\mathrm{n}=2)$ subcutaneously for DVT prophylaxis. Six patients who did not receive argatroban had a thrombotic complication, including 2 who subsequently had a positive PF4/heparin ELISA; although argatroban was not initiated in these patients, all heparin was discontinued immediately.

\section{Comparisons Between Groups}

Table 1 shows comparisons between the argatrobantreated and non-argatroban-treated groups. PF4/heparin OD was significantly greater in patients who received argatroban $(P<.001)$. There were no significant differences observed between the groups for $\Delta \mathrm{p}$; baseline, maximum, or minimum $\Delta \mathrm{p}$; and length of stay. Patients treated with argatroban were more likely to have a high 4Ts score $(P=.02)$. There were no significant differences between groups for age, gender, mortality, use of $\mathrm{CPB}$, or bleeding complications. Thrombotic complications before initiation of argatroban were more likely to occur in patients eventually anticoagulated with argatroban compared with those not treated with argatroban. In a separate comparison, patients treated with intravenous heparin anticoagulation $(\mathrm{n}=8)$ demonstrated no differences with respect to mortality, bleeding, or thrombotic events compared with those treated with argatroban. 
TABLE 1. Characteristics, complications, and laboratory values in cardiothoracic surgical patients in the intensive care unit treated or not treated with argatroban

\begin{tabular}{|c|c|c|}
\hline & $\begin{array}{c}\text { Argatroban- } \\
\text { treated } \\
(\mathrm{N}=47) \\
\end{array}$ & $\begin{array}{c}\text { Non-argatroban- } \\
\text { treated } \\
(\mathbf{N}=\mathbf{4 0}) \\
\end{array}$ \\
\hline $\begin{array}{l}\mathrm{PF} 4 / \text { heparin immunoassay } \\
\mathrm{OD}>0.40(\%)\end{array}$ & $37(79 \%)$ & $13(33 \%)$ \\
\hline $\begin{array}{l}\text { Mean PF4/heparin OD } \\
\text { (range) }\end{array}$ & $\begin{array}{l}1.012 \\
(0.104-3.000)^{*}\end{array}$ & $0.354(0.031-2.365)$ \\
\hline No. with P1 profile (\%) & $22(47 \%)$ & $16(40 \%)$ \\
\hline No. with P2 profile (\%) & $15(32 \%)$ & $6(15 \%)$ \\
\hline$\Delta \mathrm{p}($ range $)$ & $59(2-98)$ & $46(0-88)$ \\
\hline Minimum $\Delta \mathrm{p}$ (range) & $63(4-223)$ & $67(17-241)$ \\
\hline Maximum $\Delta \mathrm{p}$ (range) & $165(80-394)$ & $125(60-324)$ \\
\hline $\begin{array}{l}\text { Median 4Ts score (range, } \\
\text { interquartile range) } \dagger\end{array}$ & $4(0-7,2-5)$ & $2(0-6,0-3)$ \\
\hline High $(\geq 6) 4$ Ts score & $6(13 \%)$ & $1(3 \%)$ \\
\hline $\begin{array}{l}\text { Intermediate (4-5) 4Ts } \\
\text { score }\end{array}$ & $23(49 \%)$ & $8(20 \%)$ \\
\hline Low $(\leq 3) 4$ Ts score & $18(38 \%)$ & $31(78 \%)$ \\
\hline $\begin{array}{l}\text { SRA positive ( } \% \text { of total } \\
\text { SRA tests done) }\end{array}$ & $7 / 22(32 \%)$ & $1 / 7(14 \%)$ \\
\hline $\begin{array}{l}\text { Thrombotic complications } \\
\text { before argatroban initiated }\end{array}$ & $15(32 \%)^{*}$ & $6(15 \%)$ \\
\hline Bleeding complications & $3(6 \%)$ & $5(13 \%)$ \\
\hline $\begin{array}{l}\text { Thrombotic complications } \\
\text { after argatroban initiation }\end{array}$ & 5 & $\mathrm{n} / \mathrm{a}$ \\
\hline No. with on-pump surgery & $31(66 \%)$ & $27(68 \%)$ \\
\hline Mortality & $11(23 \%)$ & $8(20 \%)$ \\
\hline Length of stay in days (range) & $38(10-144)$ & $29(6-165)$ \\
\hline
\end{tabular}

\section{DISCUSSION}

In patients admitted to the cardiothoracic ICU after cardiothoracic surgery, the use of argatroban was not associated with bleeding complications. This is important because patients in an ICU setting after cardiothoracic surgery may be at risk for complications due to bleeding. We believe that argatroban is safe after cardiothoracic surgery because of the relatively short half-life, lack of adverse effects on platelets, and clearance independent of renal function. Argatroban is a potent anticoagulation agent, and the lack of increased bleeding complications in patients treated with argatroban is encouraging, with its rapid clearance perhaps important in its safety. ${ }^{21}$ Because we found no increased rate of bleeding, mortality, or length of stay in our patients treated with argatroban, the initiation of argatroban therapy immediately on suspicion of HIT should not be delayed, a conclusion consistent with American College of Chest Physicians guidelines. ${ }^{22}$

A greater likelihood of high 4Ts score among argatrobantreated patients suggests that the clinical 4Ts score, which quantifies thrombocytopenia, platelet dynamics, and thrombotic complications, is used by clinicians in determining whether a PF4/heparin ELISA test should be sent and whether argatroban therapy should be initiated. Thrombocytopenia during the first 4 postoperative days is rarely attributable to HIT because heparin administered during surgery usually does not induce significant levels of HIT antibodies until postoperative day 5 or later. ${ }^{23,24}$ However, it is evident from our study that treating physicians use the clinical 4Ts score to some extent in guiding diagnosis and treatment of HIT, although perhaps not explicitly. Of note, PF4/heparin OD and the incidence of thrombotic complications were significantly greater in patients treated with argatroban, suggesting that clinical suspicion for HIT in our cardiothoracic ICU is relatively appropriate in guiding argatroban initiation. For patients who had a low 4Ts score and neither P1 nor P2 platelet profile, we were unable to determine the clinical reasoning for sending a PF4/heparin ELISA. The decline of $\Delta \mathrm{p}$ between postoperative days 5-10 is a marker of platelet consumption resulting from antibodies that develop after cardiothoracic surgery and recognize multimeric PF4/heparin complexes on platelet surfaces. ${ }^{20,24}$ Although there was no association between $\Delta \mathrm{p}$ and argatroban treatment, $\Delta \mathrm{p}$ is a major component of the 4Ts scoring system, and as such this marker may be a compelling indicator that HIT should be clinically suspected and alternative anticoagulation begun without waiting for $\mathrm{PF} 4 /$ heparin immunoassay results. ${ }^{20,22,25}$

A primary safety concern with argatroban and other anticoagulants is major bleeding. In the cardiothoracic ICU after cardiothoracic surgery, unfractionated heparin is always the drug of choice because of short half-life, minimal effects of renal dysfunction, and reversibility. Some of the patients were receiving DVT prophylaxis with subcutaneous heparin; however, other indications for high- or low-dose intravenous anticoagulation included bridging for valve repair, recent valvular surgery, recent thrombotic complication, and a negative PF4/heparin ELISA result or history of DVT or PE. Patients who were not anticoagulated were thought to be at a higher risk for bleeding. Because the argatroban-treated and non-treated patients had similar outcomes in our study, one conclusion may be that argatroban is safe after CPB, and another may be that anticoagulation is not necessary. However, true HIT is rare, and specific risk factors other than heparin exposure remain unknown; the non-treated patients may not have been at higher risk for HIT.

In prospective, multicenter studies not limited to cardiothoracic surgical patients, argatroban significantly improved outcomes in HIT, particularly reducing new thrombosis and mortality without increasing risk of bleeding. ${ }^{12,13}$ In our study, it is unlikely that the bleeding complications seen in argatroban-treated patients were the result of argatroban itself because its half-life is 
approximately 45 minutes and the bleeding complications in all but 1 patient occurred at least 2 weeks after the cessation of argatroban, and all had a normal aPTT at the time of bleeding. In addition, none of the patients developed bleeding complications during the time period that argatroban was being administered. Our study is consistent with a previous retrospective analysis of 31 cardiothoracic surgery patients who received argatroban starting 1.7 days after surgery at doses of $0.66 \mu \mathrm{g} / \mathrm{kg} / \mathrm{min}$ over a median duration of 5.9 days. ${ }^{26}$ The investigators noted that no intracranial, intra-abdominal, or retroperitoneal bleeding events were reported in these patients and that no clear source of bleeding was identified from imaging studies. It is interesting to note that argatroban was approved without a randomized control group because only historic controls were used in approval of this drug. ${ }^{13}$ Thus, our study provides important additional evidence that early postoperative use of argatroban is well tolerated and associated with a low risk of thrombotic and bleeding events.

\section{Study Limitations}

There are several limitations to this study. First, because of the data-collection methods, we were unable to determine the dose ranges, dose adjustments, and duration of argatroban infusions. In addition, we currently use anti-Xa levels for monitoring heparin therapy with intravenous administration and were therefore unable to compare aPTT levels between argatroban-treated and non-argatrobantreated patients. Second, argatroban was withheld from some patients despite a positive PF4/heparin ELISA result, presumably because of a concern for an increased risk of bleeding. We did not have access to transfusion data and were therefore unable to corroborate this presumption or identify subclinical bleeding by quantifying transfusion requirements in our patients as previous studies have done. ${ }^{14}$ Last, the small number of patients included and the retrospective nature of this study made it difficult to explicitly evaluate safety. For these reasons, the actual efficacy of argatroban for the prevention of HIT and thrombotic complications cannot be determined from our data.

\section{CONCLUSIONS}

Standardized criteria, such as the 4Ts clinical scoring system and platelet profiles, can be used to appropriately initiate PF4/heparin antibody testing and immediately initiate argatroban. Clinicians often delay initial anticoagulation therapy on clinical suspicion of HIT because of safety concerns about bleeding. The lack of increased risk for adverse events with argatroban in the cardiothoracic ICU after surgery suggests early therapy with argatroban as a DTI should be considered. Other DTIs currently available for use include the parenteral agents lepirudin, desirudin, and bivalirudin, and the oral agent dabigatran. The results from our study support argatroban use on immediate clinical suspicion of HIT as detected by an otherwise unexplained decrease in $\Delta \mathrm{p}$ greater than $50 \%$ approximately 5 to 10 days postoperatively or thrombotic complications in all patients after cardiothoracic surgery and before confirmatory laboratory results. Additional prospective, randomized studies are needed to support our findings and to determine the relative safety of argatroban and other DTIs.

\section{References}

1. Warkentin TE, Sheppard J-AI, Horsewood P, Simpson PJ, Moore JC, Kelton JG. Impact of the patient population on the risk for heparin-induced thrombocytopenia. Blood. 2000;96:1703-8.

2. Marc T, Anne G, Pierre Louis C, Jean A, Anne-Marie V, Edith F. High incidence of anti-heparin/platelet factor 4 antibodies after cardiopulmonary bypass surgery. Br J Haematol. 1998;101:653-5.

3. Bauer TL, Arepally G, Konkle BA, Mestichelli B, Shapiro SS, Cines DB, et al. Prevalence of heparin-associated antibodies without thrombosis in patients undergoing cardiopulmonary bypass surgery. Circulation. 1997;95:1242-6.

4. Amiral J, Bridey F, Dreyfus M, Vissoc AM, Fressinaud E, Wolf M, et al. Platelet factor 4 complexed to heparin is the target for antibodies generated in heparininduced thrombocytopenia. Thromb Haemost. 1992;68:95-6.

5. Bennett-Guerrero E, Slaughter TF, White WD, Welsby IJ, Greenberg CS, ElMoalem H, et al. Preoperative anti-PF4/heparin antibody level predicts adverse outcome after cardiac surgery. J Thorac Cardiovasc Surg. 2005;130:1567-72.

6. Holmes-Gosh E. Heparin-induced thrombocytopenia and thrombosis syndrome after cardiopulmonary bypass. Am J Crit Care. 2000;9:276-8.

7. Kress DC, Aronson S, McDonald ML, Malik MI, Divgi AB, Tector AJ, et al. Positive heparin-platelet factor 4 antibody complex and cardiac surgical outcomes. Ann Thorac Surg. 2007;83:1737-43

8. Boggio LN, Oza VM. Argatroban use in heparin-induced thrombocytopenia. $E x$ pert Opin Pharmacother. 2008;9:1963-7.

9. Swan SK, Hursting MJ. The pharmacokinetics and pharmacodynamics of argatroban: effects of age, gender, and hepatic or renal dysfunction. Pharmacotherapy. 2000;20:318-29.

10. Krauel K, Fürll B, Warkentin TE, Weitschies W, Kohlmann T, Sheppard JI, et al. Heparin-induced thrombocytopenia-therapeutic concentrations of danaparoid, unlike fondaparinux and direct thrombin inhibitors, inhibit formation of platelet factor 4-heparin complexes. J Thromb Haemost. 2008;6:2160-7.

11. Swan SK, St. Peter JV, Lambrecht LJ, Hursting MJ. Comparison of anticoagulant effects and safety of argatroban and heparin in healthy subjects. Pharmacotherapy. 2000;20:756-70.

12. Lewis BE, Wallis DE, Berkowitz SD, Matthai WH, Fareed J, Walenga JM, et al. Argatroban anticoagulant therapy in patients with heparin-induced thrombocytopenia. Circulation. 2001;103:1838-43.

13. Lewis BE, Wallis DE, Leya F, Hursting MJ, Kelton JG. Argatroban anticoagulation in patients with heparin-induced thrombocytopenia. Arch Intern Med. 2003; 163:1849-56.

14. Yoon J, Yeh R, Nam K, Hoffman W, Agnihotri A, Jang I- K. Safety and efficacy of the argatroban therapy during the early post-cardiac surgery period. J Thromb Thrombolysis. 2010;30:276-80.

15. Harder S, Merz M, Klinkhardt U, Lorenz H, Koster A. Influence of argatroban on coagulation parameters in heparin-induced thrombocytopenia patients after cardiothoracic surgery. J Thromb Haemost. 2007;5:1982-4.

16. Horsewood P, Warkentin TE, Hayward CPM, Kelton JG. The epitope specificity of heparin-induced thrombocytopenia. Br J Haematol. 1996;95:161-7.

17. Bakchoul T, Giptner A, Najaoui A, Bein G, Santoso S, Sachs UJH. Prospective evaluation of PF4/heparin immunoassays for the diagnosis of heparin-induced thrombocytopenia. J Thromb Haemost. 2009;7:1260-5.

18. Pugh RN, Murray-Lyon IM, Dawson JL, Pietroni MC, Williams R. Transection of the oesophagus for bleeding oesophageal varices. Br J Surg. 1973;60:646-9.

19. Lo GK, Juhl D, Warkentin TE, Sigouin CS, Eichler P, Greinacher A. Evaluation of pretest clinical score (4 T's) for the diagnosis of heparin-induced thrombocytopenia in two clinical settings. J Thromb Haemost. 2006;4:759-65.

20. Pouplard C, May MA, Regina S, Marchand M, Fusciardi J, Gruel Y. Changes in platelet count after cardiac surgery can effectively predict the development of pathogenic heparin-dependent antibodies. Br J Haematol. 2005;128:837-41.

21. Shaheed G, Malkovska V, Mendoza J, Patel M, Rees J, Wesley R, et al. PF4 ENHANCED assay for the diagnosis of heparin-induced thrombocytopenia in complex medical and surgical patients. Crit Care Med. 2007;35:1691-5. 
22. Warkentin TE, Greinacher A, Koster A, Lincoff AM. Treatment and prevention of heparin-induced thrombocytopenia: American College of Chest Physicians Evidence-Based Clinical Practice Guidelines (8th edition). Chest. 2008;133: 340S-80S.

23. Aird WC, Mark EJ. Case records of the Massachusetts General Hospital. Weekly clinicopathological exercises. Cases 15-200. A 53-year-old man with a myocardial infarct and thromboses after coronary-artery bypass grafting. $N$ Engl J Med. 2002;346:1562-70
24. Warkentin TE, Greinacher A. Heparin-induced thrombocytopenia and cardiac surgery. Ann Thorac Surg. 2003;76:2121-31.

25. Warkentin TE. Platelet count monitoring and laboratory testing for heparin-induced thrombocytopenia. Arch Pathol Lab Med. 2002;126: 1415-23.

26. Yoon JH, Yeh RW, Nam KH, Hoffman WD, Agnihotri AK, Jang IK. Safety and efficacy of the argatroban therapy during the early post-cardiac surgery period. $J$ Thromb Thrombolysis. 2010;30:276-80. 